Revue d'histoire de l'Amérique française

REVUE D.HISTOIRE DE L'AMÉRIQUE FRANÇAISE

\title{
L'industrie de la chaussure à Montréal : 1840-1870 — le passage de l'artisanat à la fabrique
}

\section{Joanne Burgess}

Volume 31, numéro 2, septembre 1977

URI : https://id.erudit.org/iderudit/303607ar

DOI : https://doi.org/10.7202/303607ar

Aller au sommaire du numéro

Éditeur(s)

Institut d'histoire de l'Amérique française

ISSN

0035-2357 (imprimé)

1492-1383 (numérique)

Découvrir la revue

Citer cet article

Burgess, J. (1977). L'industrie de la chaussure à Montréal : 1840-1870 — le passage de l'artisanat à la fabrique. Revue d'histoire de l'Amérique française, 31(2), 187-210. https://doi.org/10.7202/303607ar d'utilisation que vous pouvez consulter en ligne.

https://apropos.erudit.org/fr/usagers/politique-dutilisation/ 


\title{
L'INDUSTRIE DE LA CHAUSSURE À MONTRÉAL: 1840-1870 - LE PASSAGE DE L'ARTISANAT À LA FABRIQUE
}

\author{
JOANNE BURGESS \\ Groupe de recherche sur la société \\ montréalaise au 19 e siècle \\ Université du Québec à Montréal
}

\begin{abstract}
Deux grands faits élémentaires, étroitement liés l'un à l'autre, et qui se transforment l'un par l'autre, indéfiniment variés dans leurs conséquences et toujours identiques dans leur principe, dominent cette évolution tout entière: l'échange et la division du travail...

Les époques que nous distinguons dans l'histoire économique correspondent aux degrés plus ou moins marqués de ce double développement. À ce point de vue, le machinisme lui-même, si important qu'en aient été les effets, n'est qu'un phénomène d'ordre secondaire. Avant de devenir une des causes les plus puissantes qui aient agi sur les sociétés modernes, il a commencé par être la résultante et comme l'expression de ces deux phénomènes, parvenus à un moment décisif de leur évolution. C'est ce moment de crise, caractérisé par l'apparition des machines, qui définit le mieux la révolution industrielle. ${ }^{1}$
\end{abstract}

L'histoire de l'industrialisation au Québec, plus particulièrement à Montréal, au $19^{\mathrm{e}}$ siècle, est encore mal connue. Il s'agit pourtant d'un processus qui marqua profondément la société québécoise, tout comme la révolution industrielle bouleversa la vie économique, sociale et politique de l'Angleterre des $18^{\mathrm{e}}$ et $19^{\mathrm{e}}$ siècles. Nous tenterons d'en cerner le plus précisément possible certains aspects à travers l'examen d'un secteur particulier, celui de l'industrie de la chaussure.

1 Paul Mantoux, La révolution industrielle au XVIII ${ }^{e}$ siècle (Paris, Éditions Génin, 1973), 20.

RHAF, vol. $31, \mathrm{n}^{\circ} 2$ (septembre 1977) 
L'industrialisation en Europe occidentale et en Amérique correspond à l'émergence de la grande industrie devant laquelle la boutique de l'artisan et la manufacture cèdent la place. C'est d'abord la destruction d'un mode de production: l'organisation artisanale traditionnelle de la production et du travail. Elle s'opère par l'introduction de la division du travail sous des formes de plus en plus poussées, de machines-outils de plus en plus perfectionnées et finalement par l'adjonction de la machine à vapeur. C'est également la destruction des anciens rapports sociaux de production: les rapports maîtrescompagnons et maitres-apprentis. C'est le bouleversement des conditions de vie et de travail des artisans, qu'ils soient maîtres, compagnons ou apprentis. Mais la révolution industrielle c'est aussi l'émergence de nouvelles forces productives, l'apparition d'une nouvelle étape dans l'évolution d'un mode de production, le capitalisme industriel, fondé sur la fabrique et la grande production mécanisée. C'est l'émergence de deux nouvelles classes sociales, la bourgeoisie et le prolétariat industriels.

Beaucoup d'historiens ont identifié la révolution industritelle à l'émergence et à l'utilisation accrue de la machine à vapeur, événements généralement liés à l'apparition de la fabrique. Ils ont ainsi été amenés à délaisser toutes les étapes antérieures de l'industrialisation. Ils ont alors réduit un processus long et complexe à un événement brutal et ils ont créé un mythe, la vision simpliste de l'artisan indépendant, heureux, brusquement dépossédé et transplanté en usine. Les historiens canadiens et québécois n'ont pas échappé à la règle. Quant à nous, nous estimons qu'il faut situer l'industrialisation dans le contexte plus large qu'évoque Paul Mantoux ${ }^{2}$. Nous tenterons de démontrer que, dans le cas de l'industrie de la chaussure de Montréal, la réalité est beaucoup plus complexe que l'image qu'en donne le mythe. La transition de la boutique de l'artisan à la fabrique reste brutale dans ses effets mais elle s'opère progressivement, étape par étape, sur une période de temps relativement longue.

Nous n'examinerons pas ici l'ensemble des conditions qui ont rendu ces transformations possibles, pas plus que nous n'analyserons de façon très poussée les caractéristiques de ces nouvelles classes sociales. Notre étude sera centrée sur le processus d'industrialisation lui-même, sur l'évolution de l'organisation de la production et du travail pendant les années qui marquent et la transition entre l'artisanat et la grande production mécanisée. Pour l'industrie de la chaussure de

2 Ibid. 
Montréal, cette période de la transition correspond aux années 18401870 , bien que certains changements se produisent déjà au cours des décennies précédentes.

Notre étude portera sur les quatre périodes qui correspondent aux principales étapes dans l'évolution de l'industrie de la chaussure de Montréal. Il faut d'abord voir les transformations du mode de production artisanal qui précèdent le machinisme. Nous étudierons ensuite les événements qui bouleversent l'industrie de la chaussure en 1849, au moment où sont introduites les premières machinesoutils ainsi que l'utilisation systématique d'une main-d'œuvre nonqualifiée et d'une plus grande division du travail. L'étape suivante s'échelonne sur une quinzaine d'années, de 1850 à 1864 et est marquée par l'impact que présentent l'utilisation de machines plus perfectionnées et l'introduction de la vapeur sur l'organisation de la production et du travail. Vient enfin le dernier stade de cette longue évolution vers la fabrique, qui s'étend de 1864 à 1870. Nous tenterons dans chaque cas d'analyser la réaction des artisans et des travailleurs aux transformations qu'ils subissent.

Tout au long de cette période, l'industrie de la chaussure occupe une place de premier rang dans la vie économique de Montréal. Déjà en 1825, la cordonnerie vient en tête du secteur de la fabrication et elle emploie $3,8 \%$ des travailleurs de la ville ${ }^{3}$. En 1842 , les cordonniers représentent plus de $5 \%$ des chefs de ménage recensés; Montréal compte au moins 33 commerces ou boutiques de chaussures ${ }^{4}$. Dix ans plus tard, cette proportion semble s'être maintenue: les 409 bottiers et cordonniers recensés forment $4,8 \%$ des travailleurs montréalais 5 . Un observateur décrivant Montréal à l'époque ira jusqu'à dire que tout le monde était cordonnier ${ }^{6}$. Il exagérait, mais à peine. L'industrie de la chaussure prend de plus en plus d'importance au sein de l'économie de la ville pendant les années 1850 et 1860. En 1861, avec ses 1396 employés, elle occupe 5,6\% de la main-

3 Jean-Paul Bernard, Paul-André Linteau et Jean-Claude Robert, «La structure professionnelle de Montréal en 1825», RHAF, 30, 3 (déc. 1976) : 410.

4 APC, Recensement de 1842, listes nominatives; Archives du Séminaire de Québec, Fonds Verreau 22 no 53, tableaux et compilations du recensement de 1842; Montreal Directory, 1842.

5 Recensement de 1851-52, I: tableau IV.

6 McGill University, McCord Museum, Harrison Stephens Papers, Biography of the late Harrison Stephens, esq., 11: "The fact is the whole city at that time were shoemakers." 
d'œuvre de la ville ${ }^{7}$. À peine dix ans plus tard, ce pourcentage s'est accru de façon remarquable. Les travailleurs de l'industrie de la chaussure forment le groupe professionnel le plus important de l'industrie manufacturière de la ville avec $24,4 \%$ de ses effectifs, soit 5175 cordonniers $^{8}$. Le poids économique de ce secteur est plus grand encore: W. J. Patterson estime qu'en 1872, 25000 Montréalais en dépendent ${ }^{9}$. Ainsi, la cordonnerie occupe une place prépondérante dans l'industrie manufacturière de Montréal pendant toute la période que nous analyserons.

\section{L'évolution de l'industrie de la chaussure: les grandes lignes}

Le métier de cordonnier satisfait un des besoins les plus fondamentaux des hommes: on le retrouve donc, sous une forme ou une autre dans la plupart des sociétés. La forme d'organisation qu'il prend suit généralement le même cours que l'ensemble des métiers. En Nouvelle-France, l'organisation de la production des bottes et chaussures sera fortement influencée par les pratiques et les traditions françaises. On n'introduira pas ici un régime de maitrise et jurandes, mais les outils et les techniques utilisés, la nature et l'importance de l'apprentissage, les rapports maîtres-apprentis seront fondés sur l'organisation artisanale qui existait en France à la même époque. Après la Conquête, les traditions et les pratiques artisanales britanniques seront graduellement introduites au Bas-Canada et modifieront le régime existant. Au $19^{\mathrm{e}}$, siècle, la cordonnerie subira l'influence grandissante de la Grande-Bretagne et des États-Unis ${ }^{10}$.

Examinons rapidement les grandes étapes qui marquent l'évolution de l'industrie de la chaussure dans ces deux pays avant d'étudier le cas montréalais.

À l'époque où domine l'organisation artisanale de la production, la confection des chaussures est l'œuvre d'un maître-artisan, assisté de quelques compagnons et apprentis. Il travaille dans un petit atelier,

7 Recensement des Canadas, 1860-1861, I: tableau VII.

8 Jean-Claude Robert, Montréal 1821-1871, Aspects de l'urbanisation (thèse de doctorat, École des Hautes Études en sciences sociales, 1977), 287-289.

9 Ibid., 279.

10 Sur l'industrie de la chaussure et le mode de production artisanal avant 1825, voir Louise Dechêne, Habitants et marchands de Montréal au XVII ${ }^{e}$ siècle (Paris/Montréal, Plon, 1974), 392-398, 473; Peter N. Moogk, "Apprenticeship Identures: a Key to Artisan Life in New France», Canadian Historical Association Reports (1971); Jean-Pierre Hardy et David-Thiery Ruddel, Les apprentis artisans à Québec 1660-1815 (Montréal, Les Presses de l’Université du Québec, 1977), 220. 
le plus souvent rattaché à sa demeure. Les outils du cordonnier et les techniques de fabrication sont rudimentaires et n'ont guère évolué depuis plusieurs siècles:

Voici comment il procédait: D'abord, il taillait ses semelles, ses talons et ses renforts. Il taillait ses empeignes et en assemblait les différentes pièces, en les cousant.

La seconde opération consistait à monter l'empeigne sur la forme. C'est alors que la pince courbe entrait en jeu. Puis il moulait son empeigne sur la forme en la fixant avec des chevilles qu'il tenait généralement dans sa bouche et qu'il enfonçait dans la fausse semelle.

Une fois le montage terminé, le cordonnier posait la semelle soit par l'enchevillement, soit par la couture. C'est alors que l'ouvrier, armé de son alène et de son ligneul, cousait la semelle à l'empeigne...

La dernière opération était le finissage. En tenant sa chaussure solidement appuyée contre sa poitrine, le cordonnier «trimait », découpait avec son couteau le rebord de la semelle et la rasait avec un morceau de vitre ou du papier sablé pour le rendre uniforme. Il vernissait de noir ce rebord, et, avec un fer chaud, il lui donnait du reluisant. Avec son couteau, il finissait aussi les talons et les polissait de la même manière. ${ }^{11}$

Ces bottes et chaussures sont fabriquées sur commande des clients; ce type de production a été surnommé custom-work ou bespoke-work.

Avec la croissance du marché local, le maître-cordonnier passe graduellement du travail sur commande à la vente au détail, mais les deux types de production coexistent pendant un certain temps, parfois chez le même cordonnier. Le cordonnier doit maintenir un certain inventaire de bottes et chaussures et a besoin d'un investissement plus considérable en capital circulant, pour l'achat de matières premières, pour payer les salaires de ses employés et pour l'entreposage. Cette montée de la production pour vente au détail amène l'instauration du travail à domicile et une première division technique du travail ${ }^{12}$. À ce stade, la fabrication des chaussures est divisée en trois étapes, chacune accomplie par un travailleur différent qui se

11 A. Jobin, La petite histoire de Québec (Institut Jean Bosco, 1948), 153-154; on pourrait aussi consulter Blanche Evans Hazard, The Organization of the Boot and Shoe Industry in Massachusetts before 1875 (reprints of Economic Classics, New York, Augustus M. Kelley, Publishers, 1969), 4.

12 En Angleterre, le travail à domicile dans l'industrie de la chaussure prend de l'ampleur au 18 e siècle, au moment où les organisations de métiers ne sont plus 
spécialise dans cette tâche. Ainsi, certains compagnons-cordonniers découpent le cuir (on le dénomme clicker), d'autres cousent les tiges des bottes et les empeignes des chaussures (le closer) et d'autres joignent l'empeigne ou la tige à la semelle (le maker). Le travail sur l'empeigne des chaussures est progressivement réservé aux femmes ${ }^{13}$.

En Angleterre et aux États-Unis, à la production industrielle axée sur le marché local et contrôlée par un maître-cordonnier qui vend au détail, succède la production pour un marché plus vaste: le vendeur au détail se transforme en vendeur en gros. Le marchandcordonnier qui désire produire pour vendre en gros aura besoin de beaucoup plus de capitaux, il aura à maintenir un inventaire plus considérable et devra, pour disposer de quantités suffisantes de chaussures, engager un plus grand nombre de compagnonscordonniers. Pour ce marché plus vaste, le marchand-cordonnier fournira habituellement un produit de moindre qualité qu'il aura payé moins cher au compagnon-cordonnier. On assiste donc à l'émergence d'une production «en gros» qui ne prend pas immédiatement toute la place occupée par la production sur commande et pour vente au détail mais leur livre une concurrence acharnée. Cette concurrence se manifeste avec plus d'acuité au niveau des salaires et des conditions de travail des compagnons ${ }^{14}$.

L'introduction de la production pour vente en gros sera rapidement suivie par l'introduction d'une main-d'œuvre non-qualifiée, d'une division plus poussée du travail et du sweating-system, ainsi que par l'introduction des premières machines-outils. Marx souligne l'importance de la machine-outil dans le processus d'industrialisation:

La machine-outil est donc un simple mécanisme qui, ayant reçu le mouvement convenable, exécute avec ses instruments les mêmes opérations que le travailleur exécutait auparavant avec

en mesure d'y résister. Cependant, il ne faut pas confondre le honourable trade qui utilise le travail d'artisans spécialisés travaillant à domicile et le dishonourable ou slop trade qui utilise des travailleurs moins qualifiés et encourage le sweating system.

13 Pour l'Angleterre, voir E. P. Thompson et Eileen Yeo, éd. The Unknown Mayhew (Penguin Books, 1973), 274-336; pour les États-Unis, voir John R. Commons, "American Shoemakers 1648-1895, a Sketch of Industrial Evolution», Quarterly Journal of Economics, 24 (nov. 1909): 39-81; Blanche Evans Hazard, op. cit., 3-96; Edith Abbott, Women in Industry (New York, Arno \& the New York Times, 1969), 148-185.

14 Voir surtout The Unknown Mayhew pour les conditions de travail des cordonniers à Londres et Norman Ware, "Assault on the Artisan Life Style: the Case of New England Shoemakers » dans Many Pasts : Readings in American Social History, 1600-1876, Herbert G. Gutman et Gregory S. Kealey, éd., (Englewood Cliffs, Prentice Hall, Inc, 1973), I: 233-240, pour les États-Unis. 
des instruments pareils. Dès que l'instrument, sorti de la main de l'homme, est manié par un mécanisme, la machine-outil a pris la place du simple outil. Une révolution s'est accomplie alors même que l'homme reste le moteur. Le nombre des outils avec lesquels l'homme peut opérer en même temps est limité par le nombre de ses propres organes... Le nombre d'outils qu'une même machine d'opération met en jeu simultanément est donc de prime abord émancipé de la limite organique que ne pouvait dépasser l'outil manuel. ${ }^{15}$

La machine-outil accomplit le geste que l'homme accomplissait avec son outil; l'homme ne sert plus qu'à fournir l'énergie qui actionne la machine. Après 1840, une série de machines-outils bouleversent les techniques de fabrication des chaussures, affectant tour à tour la préparation et la coupe du cuir, la piqûre des empeignes et des tiges (closing ou binding), et les opérations de montage ${ }^{16}$ (making ou bottoming). Leur introduction et leur utilisation en conjonction avec la vapeur amèneront l'ère de la fabrique et de la grande production mécanisée ${ }^{17}$.

\section{L'industrie de la chaussure à Montréal avant le machinisme}

Étudions maintenant le cas de l'industrie de la chaussure au Bas-Canada, et plus particulièrement à Montréal. L'organisation artisanale du travail et de la production prédomine dans cette industrie jusqu'au premier quart du $\mathrm{XIX}^{\mathrm{e}}$ siècle; après 1830 son importance décroît mais demeure considérable jusqu'au milieu du siècle. La transition de la production sur commande à la production pour vente au détail se produit à Montréal avant 1825 ; l'annonce suivante publiée en 1824 en est un indice typique:

John Chalmer, Boot Maker, [..] will constantly have an assortment of Boots and Shoes for Sale. As they are made up under his own inspection, of the best materials and workmanship, he can with confidence recommend them to his customer ${ }^{18}$.

15 Karl Marx, Le Capital, livre I (trad. de J. Roy, chronologie et avertissement par Louis Althusser, Paris, Garnier-Flammarion, 1969), 272-273.

16 Nous utilisons le terme montage dans son sens le plus large: «Les diverses parties de la chaussure préparées isolément, on les assemble par l'opération de montage. Il s'agit de tendre l'empeigne et de la fixer sur la semelle. » La Grande Encyclopédie (Paris, Société Anonyme de la Grande Encyclopédie, 1886-1902), XII : 954-955.

17 Pour l'évolution technologique dans l'industrie de la chaussure, on pourra consulter le Twelfth Census of the United States, 1900, IX: 754-758; William B. Rice, "The Boot \& Shoe Trade" dans One Hundred Years of American Commerce (Chauncey M. Depew, ed., New York, Greenwood Press, 1968), II: 566-567.

18 Montreal Gazette, ler septembre 1824. 
À peine quelques années plus tard, nous trouvons des indices de l'existence d'une production considérable de bottes et chaussures pour vente en gros, comme en témoigne l'annonce suivante publiée en 1833 :

Wholesale Boot \& Shoe Warehouse. The subscriber begs leave to inform his customers, and the wholesale purchasers in general, that he has on hand upwards of eight thousand pairs of Ladies' and Gentlemen's Boots \& Shoes, of the best quality. ${ }^{19}$

Le marchand-cordonnier s'adresse à une clientèle particulière: les marchands de campagne et autres détaillants qui s'approvisionnent en gros.

Ainsi, à Montréal, ces deux stades se succèdent très rapidement. Plusieurs facteurs expliqueraient cette situation. D'une part, la très forte natalité canadienne-française à cette époque multiplie rapidement les effectifs du Bas-Canada et augmente l'importance du marché régional. L'arrivée de Loyalistes et d'immigrants britanniques qui s'établissent au Haut-Canada à la même époque ouvrira un nouveau marché à la production industrielle montréalaise. D'autre part, cette immigration amène au pays des maîtres-artisans qui sauront profiter de ce marché et introduiront des techniques de production utilisées en Grande-Bretagne. Des maîtres-artisans déjà installés au pays suivront cet exemple. De plus, l'immigration de compagnons-cordonniers venus de la Grande-Bretagne et de l'Irlande permettra d'augmenter les effectifs de l'industrie et, en même temps, la production de bottes et chaussures au Canada. Ainsi, dès le début des années 1830, des observateurs notent que les producteurs canadiens ont supplanté les importations britanniques et assurent l'approvisionnement du marché canadien:

In consequence of the great number of shoemakers who settle in Quebec and Montreal, and the low prices they get for their labour in winter, added to the cheapness of Canadian and United States leather, these articles can be manufactured fully as cheap here as in Great Britain or Ireland, which has reduced the importation to a mere trifle. ${ }^{20}$

À Montréal, l'émergence de la production destinée à la vente en gros, que certains identifient à la naissance de la cordonnerie moderne, se produit vers 1825 . Le titre de pionnier est contesté, certains l'accor-

19 The Vindicator, 26 avril 1833.

20 Quebec Gazette, 30 décembre 1831. 
dant à Joshua ou à Alexander Bell, d'autres à William Galt ${ }^{21}$. Nous proposons un troisième candidat, la société Stephen Field \& Co., marchands de cuir et de chaussures (parfois aussi de beurre et de porc). Cette dernière fait publier l'annonce suivante en 1824: «Wanted 30 journeymen shoemakers acquainted with making peg shoes. ${ }^{22}$ Le nombre très élevé de compagnons engagés nous porte à croire qu'il s'agit là d'une production qui sera vendue en gros et dont le volume est important.

D'après la fréquence des annonces parues dans les journaux montréalais pendant les années 1830 , il semble que le nombre de maîtres-cordonniers vendant des chaussures en gros augmente mais que ceux qui vendent au détail conservent une place importante. C'est à ce moment que paraît se manifester pour la première fois, non seulement à Montréal mais aussi à Québec, à Toronto et à Kingston, une opposition entre les intérêts des compagnonscordonniers et ceux des maîtres-cordonniers. Cette opposition, il nous semble, ne peut que correspondre à la fin de la primauté de l'organisation artisanale traditionnelle. Nous n'avons pu vérifier si le travail à domicile existait à Montréal ou dans d'autres villes à cette époque et quel était le degré de division du travail qui y prévalait. Toutefois, les conflits qui opposent maîtres et compagnonscordonniers nous amènent à formuler cette hypothèse ${ }^{23}$.

À Montréal, nous trouvons des signes d'une association de compagnons-cordonniers pour la première fois en $1830 .{ }^{24}$ Elle est de nouveau active en 1834 :

To the Journeymen Cordwainers of Montreal of all Denominations -

21 Voir F. W. Terrill, A Chronology of Montreal and of Canada From A.D. 1752 to A.D. 1893. Including Commercial Statistics, Historical Sketches of Commercial Corporation and Firms and Advertisements Together With Calendars for Every Year from A.D. 1752 to A.D. 1925 (Montreal, Lovell, 1893), 96, repris par Raoul Blanchard, Montréal, esquisse de géographie urbaine (Grenoble, Imprimerie Allier, 1947), 56, 93; voir aussi la conférence de E. F. Ames, "The Boot \& Shoe Industry», Montreal Herald, 15 janvier 1884; pour plus de renseignements sur Joshua Bell, voir JeanClaude Robert, «Joshua Bell», Dictionnaire Biographique du Canada.

22 Montreal Gazette, 9 octobre 1824.

23 E. F. Ames décrit le travail à domicile et la division du travail qui existent à Montréal avant l'introduction de la machine dans l'industrie de la chaussure. Il ne précise ni comment, ni à quel moment, ils furent inaugurés.

24 Charles Lipton, The Trade Union Movement in Canada (2 éd., Montreal, Canadian Social Publications Limited, 1968), 3. 
We the undersigned, acting in behalf of the General Trade and considering that the unprecedented depression under which we now labour, can only be removed by a cordial cooperation and unanimity of purpose, in the different branches of the Trade, do now most earnestly call upon our fellow mechanics, to meet at the house of $[\ldots]$ to adopt such measures as may seem best calculated for their mutual protection and promoting the general interests of the trade. ${ }^{25}$

Cet avis est signé par 12 compagnons-cordonniers, dont 3 Canadiens français. Il en ressort qu'ils connaissent à ce moment une période très difficile et décident de s'unir pour se protéger. Nous ne connaissons pas l'efficacité de cette association; toutefois dans un avis publié par une douzaine de maîtres-cordonniers quelques mois plus tard, ces derniers exigent d'être payés comptant, invoquant les hauts salaires versés aux compagnons: "the difficulty and expense attending the collection of small accounts, and the small profits, owing to the high salaries paid to Journeymen $"{ }^{26}$

À Québec en 1835, les compagnons-cordonniers s'unissent pour former la Quebec Journeymen Shoemakers' Society qui se donne comme objectifs, "supporting the Trade, and relieving the sick belonging to the same." Il est cependant intéressant de noter qu'un maître-cordonnier dénonce l'association dans les termes suivants:

they have endeavoured in more than one instance to control their employers, by threatening a strike on them, unless they would accede to their wishes, and actually carried it into effect with me, because I would not discharge men who did not feel free to join them. ${ }^{27}$

À Toronto les compagnons-cordonniers font la grève contre les maîtres en $1830^{28}$ et en $1835^{29}$. À Kingston au Haut-Canada, nous trouvons un autre exemple de l'organisation des compagnonscordonniers pour la défense de leurs intérêts ${ }^{30}$.

Nous croyons que ces manifestations de militantisme chez les compagnons-cordonniers de plusieurs villes sont liées aux modifica-

25 The Vindicator, 4 avril 1834.

26 The Vindicator, 10 juin 1834.

27 Quebec Mercury, 25, 28 et 30 avril 1835.

28 Charles Lipton, op. cit., 6.

29 Kingston Chronicle, 16 septembre et 17 octobre 1835.

30 Le Montreal Courier du 25 septembre 1838 contient l'annonce suivante: "Journeymen Shoemakers Wanted. 40 or 50 good workmen will find constant employment and good wages, on application to the Master Shoemakers of Kingston. „ Ce type d'annonce est habituellement l'indice d'un conflit de travail. 
tions de leurs conditions de travail qui surviennent lorsque la production pour vente au détail et en gros remplace la confection sur commande.

Pendant les années 1840 , les transformations amorcées au cours des vingt années antérieures s'amplifient. Les marchands-cordonniers produisant pour vendre en gros se différencient de plus en plus nettement des maîtres-cordonniers et des cordonniers vendant au détail. Cette différenciation se manifeste avec le plus d'acuité au niveau des capitaux requis pour se maintenir en affaires ${ }^{31}$. Il est de plus en plus difficile à un simple compagnon d'avoir accès au crédit requis pour devenir marchand-cordonnier. Le sort de la vaste majorité des cordonniers sera dès lors d'être compagnons pour toute leur vie.

À cette même époque, nous trouvons ce que nous croyons être un indice de l'existence du travail à domicile dans l'industrie de la chaussure de Montréal. En 1846, Martin Thornton, un maîtrecordonnier tenant boutique, décide d'abandonner les affaires. L'acte de vente fournit l'inventaire du travail en cours:

23 pairs wrought boot legs

29 pairs Fronts and 8 pairs outclosing

12 paris Gators outmaking

7 pairs wrought suff ciff (sic) morocco patent ${ }^{32}$

Deux expressions retiennent notre attention: outclosing et outmaking. Nous y retrouvons la terminologie utilisée par Henry Mayhew à Londres en 1850 pour désigner deux aspects de la confection d'une chaussure $^{33}$. Le terme closing s'applique à l'action de coudre ensemble les pièces de la tige ou de l'empeigne, tandis que making signifie coudre la semelle à l'empeigne ou à la tige. La présence du préfixe out indiquerait, à notre avis, que ces étapes de la fabrication sont accomplies à l'extérieur de la boutique de Thornton.

L'industrie de la chaussure n'est plus au stade artisanal traditionnel à Montréal à la veille de la deuxième moitié du $\mathrm{XIX}^{\mathrm{e}}$ siècle. L'organisation de la production, c'est-à-dire le degré de division du travail et les rapports existant entre les producteurs et les moyens de

31 Voir Joanne Burgess, L'industrie de la chaussure à Montréal, 1840 à 1870 , de l'artisanat à la fabrique (thèse de M.A., Université du Québec à Montréal, 1977), chap. 1.

32 Archives judiciaires de Montréal, Greffe du notaire John Helder Isaacson, no 88,24 avril 1846 .

33 E. P. Thompson et Eileen Yeo, éd., op. cit., 279-280. 
production, a subi certaines transformations qui ont modifié profondément les conditions de travail de la plupart des cordonniers, employeurs comme employés. L'atelier du maître-artisan n'est plus le lieu unique de fabrication et de vente des chaussures. De plus en plus la boutique ne sert qu'à la coupe du cuir, à l'entreposage des matières premières et du produit fini et à la vente. La confection des bottes et des chaussures, divisée en deux étapes distinctes, se fait à l'extérieur de la boutique du marchand-cordonnier. Ces transformations sont le résultat de l'élargissement du marché pour les chaussures et de l'action de certains maitres-cordonniers qui adaptent la production en conséquence. Toutes ces transformations précèdent le machinisme.

\section{L'industrie de la chaussure à Montréal en 1849 : introduction des premiè- res machines-outils et d'une division plus poussée du travail}

Jusqu'en 1849, l'industrie de la chaussure de Montréal évolue graduellement. Si certaines modifications importantes se sont produites, plusieurs des caractéristiques du mode de production artisanal persistent. Les techniques de fabrication et les outils du cordonnier sont inchangés et le travail de confection est accompli par un artisan qui connait les différents aspects de son métier et qui a subi un long apprentissage. En 1849, cette situation sera transformée brusquement. Les responsables de ce changement sont deux Américains, agissant en société sous le nom de Brown \& Childs.

Champion Brown et William Sullivan Childs s'établissent à Montréal vers 1845; originaires du Massachusetts, ils ont à ce moment 23 et 29 ans respectivement ${ }^{34}$. Ils semblent avoir débuté comme importateurs pour se transformer très rapidement en manufacturiers. Dès leur arrivée à Montréal ils se montrent très entreprenants et signifient leur intention de vendre au plus bas prix possible:

their previous facilities for manufacturing being greatly improved, they will furnish Boots and Shoes at greatly Reduced Prices... One fact to be remembered is, there is more money saved in purchasing at 133 Notre-Dame Street than at any other place. ${ }^{35}$

Cet objectif les amènera à introduire à Montréal de nouvelles techniques de production leur permettant de réduire davantage leurs prix; c'est ce qu'ils font au printemps de 1849: «From the introduc-

34 APC, Recensement de 1861, Listes nominatives, Ville de Montréal, fiches no 4401 et $\mathbf{8 8 0 9}$.

35 Montreal Herald, 4 février 1847. 
tion of Labor-Saving machinery, they are enabled to reduce their former prices 10 per cent. ${ }^{36}$

Il nous a été impossible d'identifier avec certitude la machine introduite par Brown \& Childs en 1849; l'étude de l'évolution technologique aux États-Unis nous porte à croire qu'il s'agit de la leatherrolling machine, une machine qui facilite la préparation du cuir et qui permet de faire en une minute ce qui autrefois nécessitait trente minutes d'efforts ${ }^{37}$. L'introduction d'une telle machine, augmentant les quantités de cuir prêt à être taillé et façonné, aurait créé de fortes pressions sur les étapes subséquentes de la fabrication, incitant ainsi à l'accroissement de la division du travail. Quelle que soit cette nouvelle machine, elle bouleverse les conditions de travail des compagnons-cordonniers; ceux-ci prennent rapidement conscience de ses effets néfastes et y résistent.

Au printemps de 1849 , les compagnons-cordonniers de Montréal sont regroupés au sein d'une association, la Journeyman Shoemakers' Society. Cette société a pignon sur rue et est administrée par un comité exécutif ${ }^{38}$. Ses objectifs sont à la fois ceux d'une société de secours mutuel ${ }^{39}$ et ceux d'une association ouvrière ${ }^{40}$. Le 2 avril 1849, la Société convoque les maîtres-cordonniers à une réunion pour fixer les prix des divers types de chaussures. Brown et Childs n'assistent pas à cette réunion au cours de laquelle la Société leur déclare la grève,

the Society pronounced their shop in the technical language of trades unions, a "Scab Shop", and combined to put them down, by compelling their workmen to make a strike, or to discontinue working. ${ }^{41}$

Cette grève n'est pas seulement liée à des revendications monétaires. Brown et Childs avouent qu'une délégation de membres de la société a fait des demandes très précises visant à bloquer le processus de mécanisation et de division du travail:

36 Montreal Herald, 7 mai 1849.

37 Blanche Evans Hazard, op. cit., 75-76.

38 Montreal Transcript, 9 juin 1849; Montreal Gazette, 28 octobre 1849.

39 "for the support of sick, indigent or decayed members, and such other matters as would conduce to the welfare and permanence of the trade." Montreal Gazette, 28 octobre 1849.

40 "for the purpose of regulating the prices of work to be paid to journeymen for their labour, and for protecting the masters form being swindled out of materials ", Montreal Gazette 28 octobre 1849.

41 Montreal Gazette, 28 octobre 1849. 
It is true that a deputation waited upon us to induce us to lay aside our machinery, stop the subdivision of labour, and discharge all parties not belonging to the Society; this proposition we refused to comply with. ${ }^{42}$

La grève contre Brown \& Childs dure seize semaines; pendant cette période les compagnons reçoivent l'appui de certains maîtrescordonniers qui eux aussi se sentent menacés par ces innovations technologiques. Le déroulement de ce conflit de travail révèle le haut degré d'organisation des compagnons. Dans un premier temps, la Société interdit à ses membres de travailler pour Brown \& Childs et tente d'amener leurs employés à quitter le travail:

an association of journeymen shoemakers... made a bye-law denouncing a fine of ten shillings against whomsoever, of the association, should work for B. \& C., and expulsion for repetition of such act,... at a meeting of the Association, where the Master-Shoemakers indicted were present, there was read, by the Secretary, a list of the numbers of journeymen in the employ of B. \& C., whom, when drawn or forced from that employ the masters present were willing to take... ${ }^{43}$

On organise devant le magasin de Brown \& Childs une garde: composée de membres de la Société et financée par une cotisationı obligatoire:

the members of the association provided funds to pay that guard, by levying three pence per week on each journeyman... two of the masters indicted actually stopped the money from the wages of two witnesses for the prosecution, then in their employ. ${ }^{44}$

Les membres de la Société soutiennent que l'objet de la garde est de s'assurer qu'aucun membre ne se présente chez Brown \& Childs pour travailler. Le mot d'ordre était d'éviter toute violence et s'il y en eut, ce fut, disent-ils, à l'instigation de «worthless members of the profession». D'après d'autres témoins, les tactiques des compagnons et des maîtres-cordonniers qui les appuient visent à interrompre la production chez Brown \& Childs :

those guards assaulted and insulted persons, male and female, coming out of B. \& C.'s shop with work, and pursued them to and threatened them, at their own houses...

42 Montreal Gazette, $1^{\mathrm{er}}$ novembre 1849.

43 Montreal Gazette, 28 octobre 1849.

44 Ibid. 
In this disagreable dilemma some of the hands removed to the country parishes 30 or 40 miles from town, taking quantities of work with them to make up, and were even followed to this distance, other parties congregated together into houses in town, where they lived and worked, and armed themselves for mutual protection, in which Messrs. B. \& C. assisted them... ${ }^{45}$

Nous nous sommes permis de décrire ce conflit de travail de manière détaillée parce que l'accumulation des faits montre l'acharnement des compagnons-cordonniers et la conscience qu'ils ont de l'importance de cette lutte. On ne peut sous-estimer l'impact de ce conflit de travail sur les opérations de Brown \& Childs. Un extrait d'une annonce qu'ils font publier en juillet 1849 est très éloquent à cet égard:

We believe it to be now comparatively safe for Journeyman to work for us, as the Watch is not now on more than two-thirds of the time; and there as not been, to our knowledge, a man or child waylaid or beaten for the last three days. ${ }^{46}$

Champion Brown et William S. Childs ne subiront pas cette situation sans riposter. Ils utilisent tous les moyens dont ils disposent pour briser la grève des compagnons-cordonniers. Ils tentent de déplacer la production pour éviter la surveillance étroite des grévistes. Ils recrutent une nouvelle main-d'œuvre pour remplacer les artisans en grève ${ }^{47}$. Ils importent des chaussures de Boston afin de pouvoir remplir leurs commandes ${ }^{48}$. De plus, ils passent à l'offensive. Dans un premier temps, ils ont recours aux forces de l'ordre pour combattre les lignes de piquetage qui sont montées devant leurs portes ${ }^{49}$. Par la suite, ils réussissent à obtenir des renseignements de certains membres de la Société sur les activités de celle-ci et ils obtiennent que des poursuites au criminel soient intentées contre vingt-deux compagnons et maîtres-cordonniers pour conspiration. Ces procédures judiciaires semblent mettre fin à la grève. Le procès se tient lors des assises d'octobre et le verdict du jury est favorable aux compagnons et aux maîtres-cordonniers ${ }^{50}$.

Quelques mois plus tard, les compagnons et maîtres-cordonniers de Montréal fêtent leur victoire et présentent une coupe d'argent à

\footnotetext{
45 Ibid.

46 Montreal Herald, 3 juillet 1849.

47 « 500 journeymen wanted», Montreal Herald, 7 mai 1849.

48 Montreal Herald, 3 juillet 1849.

49 Montreal Herald, 26 avril 1849.

so Montreal Gazette, 28 octobre 1849.
} 
l'avocat qui les a représentés lors du procès ${ }^{51}$. Cependant, s'ils ont remporté une victoire sur le plan juridique, les artisans et surtout les compagnons-cordonniers ont, au niveau économique, nettement perdu leur grève. Dès janvier 1850 , des indices nous montrent que Brown \& Childs poursuivent leurs efforts pour réduire leurs coûts de production par l'utilisation systématique d'une main-d'œuvre moins qualifiée et par une division plus poussée du travail:

Journeymen Shoemakers who are desirous of obtaining a Fair Remuneration For Their Labour, and are also anxious to rescue themselves from the grinding system pursued towards them, (especially by a certain Slop Shop in this city), are urgently requested to attend, a business connected with their welfare will be submitted to the meeting. ${ }^{52}$

L'enjeu du conflit qui bouleverse l'industrie de la chaussure de Montréal en 1849 est très clair. Les compagnons, les maîtrescordonniers et Brown \& Childs savent que c'est toute l'organisation de la production qui est remise en question par ces derniers lorsqu'ils affirment leurs objectifs,

confiding in their rights as English subjects, to use all means for producing most with least labor and expense ; and disregarding the combination of competitors forcibly to prevent them... ${ }^{53}$

À partir de ce moment, les compagnons-cordonniers devront progressivement céder la place aux travailleurs non-qualifiés et aux machines. Les maîtres-cordonniers auront à s'adapter à ces nouvelles techniques de production ou à subir les effets de leur concurrence destructrice. Brown \& Childs sont les vainqueurs définitifs de ce conflit et leur victoire est celle du capitalisme industriel naissant.

\section{Introduction de nouvelles machines-outils et la longue marche vers la fabrique}

Quand débute la deuxième moitié du $19 \mathrm{e}$ siècle, l'industrie de la chaussure de Montréal a déjà connu des perturbations majeures liées, d'une part, à l'introduction d'une machine qui affecte la préparation et la coupe du cuir et, d'autre part, à l'utilisation de travailleurs nonqualifiés pour confectionner des chaussures avec ce cuir. Cependant, si l'organisation du travail dans la fabrication est affectée, les outils

\footnotetext{
$51 \quad$ Montreal Gazette, 11 septembre 1850.

52 Montreal Transcript, 10 janvier 1850.

53 Montreal Herald, 7 mai 1849.
} 
traditionnels avec lesquels le cordonnier transforme les pièces de cuir en une chaussure ou une botte ont été très peu touchés. La première machine qui affectera sensiblement la confection proprement dite est la machine à coudre ${ }^{54}$.

Celle-ci fait son apparition à Montréal vers $1851^{55}$, mais elle ne semble pas avoir été immédiatement adoptée par les marchandscordonniers de la ville. Une brochure publiée en 1856 fait état d'une entreprise de chaussure qui utilise huit machines à coudre ${ }^{56}$. Dans les années suivantes, cette machine deviendra de plus en plus répandue.

L'introduction de la machine à coudre bouleverse une étape de la confection des chaussures, la piqûre des empeignes et des tiges (binding ou closing), qui était habituellement confiée aux femmes. Elle aura pour conséquence la disparition progressive du travail féminin à domicile et son intégration à l'atelier central ${ }^{57}$. Ainsi, cette machine et d'autres innovations qui se succèdent à la fin des années 1850 accroissent l'importance dans la production des chaussures de l'atelier central, de plus en plus souvent désigné sous le nom de factory. Cependant, sur le plan technique, une autre étape du travail, le montage (making ou bottoming), n'est pas touché par ces transformations.

Une des premières conséquences de la mécanisation de certaines étapes de la production est d'augmenter le nombre de travailleurs à domicile impliqués dans les autres phases de la production, et ce, jusqu'au moment où ils seront à leur tour déplacés par des machines. Ce résultat du processus d'industrialisation, contradictoire en apparence, est analysé par l'historien anglais E. P. Thompson,

The numbers employed in the outwork industries multiplied enormously between 1780-1830; and very often steam and the factory were the multipliers. It was the mills which spun the yarn and the foundries which made the nail-rod upon which the outworkers were employed. Ideology may wish to exalt one and

54 La machine à coudre fut inventée par Elias Howe en 1845 et son apport à la fabrication des bottes et chaussures fut rapidement découvert. Aux États-Unis elle est utilisée dès 1852 pour la piqûre des tiges et empeignes, et vers 1855 son utilisation est très répandue. Voir Edith Abbott, op. cit., 164; Blanche Evans Hazard, op. cit., 93-96.

55 Montreal Herald, $1^{\mathrm{er}}$ mars 1851 et Montreal Gazette, 17 juin 1851.

56 Montreal in 1856. A Sketch Prepared for the Celebration of the Opening of the Grand Trunk Railway of Canada (Montreal John Lovell, 1856), 45.

57 Edith Abbott, op. cit., 167. 
decry the other, but facts must lead us to say that each was a complementary component of a single process... Indeed, we may say that large-scale sweated outwork was as intrinsic to this revolution as was factory and steam. ${ }^{58}$

Dans l'industrie de la chaussure, la couture de la semelle à l'empeigne continue d'être faite à la main avec les outils traditionnels. Avec la mécanisation des étapes antérieures et surtout avec l'introduction de la vapeur au début des années 1860 , la production de pièces de cuir et d'empeignes augmente très rapidement, accroissant en même temps la pression sur le travail manuel. Cette dernière amène une croissance des effectifs à l'extérieur de l'usine et incite les entrepreneurs à pousser encore plus loin la division du travail. Le travail manuel à l'extérieur de l'usine, pour le compte du capitaliste, prend de plus en plus d'ampleur jusqu'à l'introduction de la McKay sole sewing machine, inventée en 1862. Celle-ci permet de coudre la semelle à l'empeigne et réduit considérablement le temps requis pour accomplir cette opération. En effet, pendant que le cordonnier pose une semelle, la machine en pose quatre-vingts 59 . La dernière étape de la fabrication pourra alors être intégrée à l'usine. À partir de ce moment, nous retrouvons la grande production mécanisée dans la chaussure et nous pouvons parler de l'existence de la fabrique. Il y aura certes des raffinements technologiques ultérieurs; leur analyse dépasserait le cadre de cette étude.

Examinons maintenant comment et à quel moment ces dernières étapes dans l'évolution vers la fabrique sont franchies à Montréal. Un ouvrage publié en 1864 nous fournit des données très précises concernant la condition de cinq entreprises de chaussure à Montréal ${ }^{60}$. En étudiant en profondeur chaque étape de la fabrication, nous pouvons mieux saisir l'état des forces productives, le niveau de perfectionnement de l'outillage et le degré de division du travail.

Voyons d'abord les procédés de préparation et de coupe du cuir. Dans toutes les entreprises, les machines-outils utilisées sont essen-

58 E. P. Thompson, The Making of the English Working Class (Penguin Books, 1974), 288-289.

59 Don. D. Lescohier, The Knights of St. Crispin, 1867-1874 (Madison, University of Wisconsin Press, 1910), 18.

60 Montreal Business Sketches with a description of the City of Montreal. Its Public Buildings, and Places of Interest, and the Grand Trunk Works at Pointe St. Charles, Victoria Bridge, etc, etc. Prepared and Published by the Canadian Railway Advertising Company (Montreal, M. Longmore \& Co, 1864), 69-75, 84-88, 95-96, 106107, 123-124. 
tiellement les mêmes, mais Brown \& Childs sont les seuls à en posséder qui soient actionnées par la vapeur. Dans cette entreprise, on a regroupé ces étapes préalables dans un seul édifice connu sous le nom de steam factory et quelque peu éloigné du bâtiment principal où se fait l'assemblage; les entreprises concurrentes n'ayant pas la vapeur concentrent toutes les opérations dans un même édifice. L'atelier servant à la préparation et à la coupe du cuir utilise des machines pour découper, rouler, dédoubler et préparer le cuir ainsi que d'autres qui taillent les semelles et forment les talons. Le travail y semble réservé exclusivement aux hommes ${ }^{61}$.

La deuxième étape de la fabrication, la couture des pièces de l'empeigne, s'accomplit aussi en usine mais ces machines ne semblent pas être mues par la vapeur. Dans ce service nous retrouvons surtout des machines à coudre mais il y a aussi certaines machines plus spécialisées. Ce travail occupe la quasi-totalité des femmes travaillant en usine ${ }^{62}$.

Une fois l'empeigne cousue, il ne reste plus qu'à la joindre à la semelle, soit avec une cheville (peg) ou avec du fil. On retrouve dans plusieurs entreprises une salle de chevillage (pegging room) où des appareils clouent la semelle à l'empeigne. La description des usines, pourtant très minutieuse, n'indique jamais la présence de cordonniers en train de coudre à la main la semelle à l'empeigne d'une chaussure. Cette situation, liée au nombre important d'employés travaillant à l'extérieur de l'usine - environ 350 dans le cas de Brown \& Childs, 150 chez W. McBain \& Co. et 300 chez Scholes \& Ames - indique décidément que cette étape de la fabrication est encore accomplie par des cordonniers travaillant à domicile ou en petits ateliers à l'extérieur de l'usine. L'importance numérique de cette dernière catégorie de travailleurs n'est pas à négliger. Chez Scholes \& Ames, ils sont trois fois plus nombreux que les travailleurs en usine; chez W. McBain \& Co. ils sont 150 des 250 employés, tandis que chez Brown \& Childs ils représentent plus de la moitié de la force de travail ${ }^{63}$.

Il est impossible d'établir de façon définitive quel est le degré de spécialisation et de formation de ces cordonniers à domicile. Nous ne savons pas si ce sont des artisans capables de confectionner 
toute une chaussure même si en l'occurence il n'en font qu'une partie. Nous ne savons rien des conditions dans lesquelles œuvrent ces cordonniers hors-usine: sont-ils victimes du sweating system ou jouissent-ils encore de cette liberté dans l'emploi du temps et dans l'organisation du travail qui caractérise la production artisanale? Bref, est-ce que le cordonnier travaillant à domicile à l'époque de la manufacture et de la fabrique est encore un artisan?

\section{L'avènement de la fabrique}

Au début de 1864, l'industrie de la chaussure à Montréal est donc à un tournant décisif de son évolution. Nous sommes à une époque où la manufacture domine. La plus grande part des opérations a été transférée à l'usine: nous y retrouvons une division du travail très poussée, des machines-outils relativement perfectionnées mais qui utilisent surtout la force motrice humaine. Dans les mois qui suivent, cette industrie franchira une autre étape en atteignant le stade de la fabrique et de la grande production mécanisée. Deux éléments sont au cour de cette transformation: l'introduction de la machine à vapeur et son application à toutes les étapes de la fabrication, ainsi que l'intégration dans l'usine des dernières étapes de la production, le montage et la couture de la semelle à l'empeigne (bottoming).

Ainsi, la machine à vapeur commence dès 1864 à remplacer la force humaine dans l'industrie de la chaussure. Elle sert d'abord à actionner les machines affectées à la préparation et à la coupe du cuir mais son utilisation s'étend très rapidement à d'autres étapes de la fabrication. Dès juillet 1864, l'entreprise Ames, Millard \& Co. emploie des machines à vapeur dans les opérations de chevillage et de couture. ${ }^{64}$ De plus en plus d'entreprises suivront cet exemple.

D'autre part, on constate aussi dès 1864 les débuts de l'intégration à l'usine de la dernière étape de la fabrication. Encore une fois c'est l'entreprise de Ames, Millard \& Co. qui prend les devants:

There is in the same room a new machine capable of sewing on two hundred pairs of soles per day, but it has not yet been fitted together. ${ }^{65}$

Le rapport de W. J. Patterson sur l'activité économique à Montréal en 1864 fait état de trois sole-leather sewing machines en service;

64 Montreal Gazette, 27 juillet 1864.

65 Ibid. 
ces machines semblent correspondre à la McKay sole-sewing machine. En 1867 le nombre de ces machines passe à quinze et en 1872 on en compte trente dans la ville de Montréal ${ }^{66}$. Cette progression rapide aura un impact considérable sur l'organisation du travail dans l'industrie et sur les conditions de travail d'un grand nombre de cordonniers. L'introduction de cette machine et l'utilisation de la vapeur pour l'actionner marquent vraiment le début de l'ère de la grande production industrielle et présagent la fin du travail à domicile dans l'industrie de la chaussure de Montréal.

Une analyse rapide du recensement industriel de 1871 pour la ville de Montréal nous permet de mesurer l'influence de ces transformations sur la production et sur la structure de l'industrie de la chaussure de la ville. En 1871, 117 entreprises de la chaussure sont dénombrées employant 5175 cordonniers et produisant des chaussures valant $\$ 5673415$. Les dix-sept entreprises ayant une production annuelle supérieure à $\$ 100000$ ne représentent que $14,5 \%$ des établissements recensés mais emploient 78,0\% des effectifs de l'industrie (soit 4040 cordonniers) et fournissent $81,5 \%$ de la production annuelle (soit $\$ 4622000$ ). Treize de ces entreprises utilisent la vapeur ${ }^{67}$.

Le passage à la fabrique bouleverse profondément les conditions de vie et de travail des cordonniers à domicile. Nous ne sommes pas en mesure d'affirmer que leur existence avant ces innovations techniques était idyllique, mais on peut penser que l'adaptation à la discipline de l'usine est très pénible. Cependant, le problème plus fondamental posé par l'introduction de la McKay sole sewing machine est celui du chômage. L'introduction antérieure d'outillage perfectionné pour tailler le cuir et assembler l'empeigne avait nécessité une expansion considérable du nombre de cordonniers travaillant à coudre la semelle à l'empeigne. Or, non seulement la nouvelle machine amène un transfert de ceux-ci à l'intérieur de l'usine, mais elle permet une économie de main-d'œuvre. Si une machine permet de coudre deux cents semelles par jour, voilà dix, vingt ou trente cordonniers sans travail. De plus, quelle habileté est requise pour la conduite de cette nouvelle machine? Le cordonnier-artisan autrefois travailleur à domicile est-il menacé non seulement par d'autres cordonniers-artisans

66 W. J. Patterson, Report on the Trade and Commerce of the City of Montreal. Compiled for the Board of Trade and the Corn Exchange Association (Montreal, 1863-1885).

67 Recensement de 1871. Ville de Montréal, recensement industriel, listes nominatives: Recensement de 1871. III: tableau XXIX. 
mais aussi par la masse des journaliers, des travailleurs non-qualifiés? Nous le croyons, mais nous connaissons mal le degré d'habileté requis pour les différentes tâches au sein de l'usine. Mais il est certain que ce surplus de main-d'œuvre permettra au propriétaire d'usine de faire encore plus d'économies: il y aura de fortes pressions à la baisse sur les salaires.

C'est dans ce contexte qu'il faut se situer pour analyser les premières organisations de défense des intérêts des ouvriers de l'industrie de la chaussure de Montréal. Dès 1868-69 apparaît à Montréal la Société des Chevaliers de Saint-Crispin; cette Société avait été fondée à Milwaukee au Wisconsin quelques années auparavant et connut une expansion fulgurante à travers les États-Unis et le Canada. ${ }^{68}$ À Montréal, l'organisation s'implante rapidement et s'impose.

L'association... est très puissante en cette ville; elle compte dans son sein presque tous les cordonniers dont le nombre est presqu'égal à celui des autres corps de métiers réunis à Montréal. ${ }^{69}$

$\mathrm{Au}$ cours de 1869, la Société organise et appuie plusieurs grèves dont la plus importante paralyse la fabrication des chaussures à Montréal pendant plus d'un mois. Lors de ces conflits de travail, les cordonniers-grévistes formulent essentiellement deux demandes. D'une part, ils s'opposent à l'embauche de travailleurs non-qualifiés et demandent que seuls soient employés des cordonniers membres de la Société. ${ }^{70}$ D'autre part, les Chevaliers de Saint-Crispin s'insurgent contre les réductions de salaires qu'ils ont subies dans le passé et demandent une augmentation afin de compenser la hausse du coût de la $v^{2} \mathrm{e}^{71}$. Ces confrontations opposent pour la première fois, à notre connaissance, travailleurs et propriétaires d'usine dans l'industrie de la chaussure de Montréal.

Nous ne voulons pas ici entreprendre une analyse détaillée des Chevaliers de Saint-Crispin: de leur organisation, de leur idéologie, de leurs effectifs, de leur évolution. Évidemment, certaines questions se posent avec plus d'acuité: quels travailleurs de la chaussure sont

68 Au sujet des Chevaliers de Saint-Crispin, voir Don. D. Lescohier, op. cit. et Greg Kealey, "Artisans Respond to Industrialism: Shoemakers, Shoe Factories and the Knights of St. Crispin in Toronto", Canadian Historical Association, Historical Papers, 1973: 137-157.

$69 \quad$ Le Pays, 11 septembre 1869.

70 Le Pays, 16 avril 1869.

71 Le Pays, 11 septembre 1869. 
membres de la Société? Dans quelle mesure leurs revendications sont-elles déterminées par leur place dans le procès de production? Malheureusement, nous ne pouvons répondre maintenant. Ce que nous avons voulu souligner c'est le lien fondamental entre l'apparition des Chevaliers de Saint-Crispin et les transformations technologiques qui bouleversent l'industrie de la chaussure et marquent le début de la grande production mécanisée.

\section{Conclusion}

Dans l'industrie de la chaussure à Montréal, c'est par un processus long et complexe s'échelonnant sur plus de trente ans, que l'artisanat se transforme et se dissout et qu'un nouveau mode de production naît.

Dans les années qui précèdent 1849 , le développement du marché régional et national amène une division plus poussée du travail et les débuts du travail à domicile. Le maître-cordonnier se transforme en marchand-fabricant vendant en gros et qui, pour mener ses affaires, requiert des sommes de plus en plus considérables de capitaux. Le compagnon-cordonnier aura beaucoup de difficultés à accumuler le capital requis pour tenir boutique et il se différencie plus nettement des maîtres, surtout de ceux-là qui ne produisent plus principalement sur commande. Les compagnons prennent conscience de leur nouvelle situation et, dès les années 1830, s'organisent pour défendre leurs intérêts face aux maîtres-cordonniers.

De 1849 à 1864 , nous assistons à l'introduction d'une série de machines-outils qui affectent surtout la coupe et la préparation du cuir et le travail sur l'empeigne de la chaussure. L'utilisation de ces machines est liée à l'introduction et l'emploi systématique de travailleurs non-qualifiés, ce qui n'est possible qu'avec une division encore plus poussée du travail. Pendant cette période, l'atelier central prend de plus en plus d'importance au fur et à mesure que différentes étapes de la production y sont intégrées et que la vapeur commence à remplacer l'homme comme source d'énergie. Les compagnonscordonniers, appuyés de certains maîtres, s'opposent par une longue grève lorsque la machine-outil et le travailleur non-qualifié viennent bouleverser leur monde. Les agents du mode de production artisanal, maitres et compagnons, résistent aux premières attaques du capitalisme industriel, mais leur opposition est vaine. Pour survivre, les maîtres-artisans devront à leur tour, pendant les années 1850 , adopter ces nouvelles méthodes de production. 
Enfin, de 1864 à 1871 , la dernière étape est franchie et la fabrique commence à dominer l'industrie de la chaussure de Montréal. La machine à vapeur prend de plus en plus d'importance dans la production et l'introduction de la McKay sole sewing machine rend possible l'intégration de la dernière étape de la confection des chaussures. Avec son introduction s'amorce le déclin du travail à domicile. Il est significatif de noter que c'est peu après le début de l'ère de la fabrique que les cordonniers de Montréal se regroupent au sein des Chevaliers de Saint-Crispin. À ce moment, nous assistons aux premiers conflits opposant prolétaires et capitalistes dans l'industrie de la chaussure de Montréal.

Ainsi, nous avons tenté de démontrer comment s'est opérée, dans une industrie de Montréal, la transition de l'artisanat à la fabrique. Si la fabrique marque le début d'une ère nouvelle, elle est en même temps l'aboutissement d'un long processus. L'organisation artisanale du travail et de la production sera profondément altérée dès les années 1820 par la réorganisation du travail et de la production ên répoñuse à l'extension du marché. Les machines-outils et la machine à vapeur transformeront davantage un monde qui, à Montréal, n'est déjà plus celui de l'artisan traditionnel. Nous ne pouvons donc pas souscrire à la vision qui trop souvent nous a été proposée d'un artisan brusquement transplanté en usine. Il est néanmoins vrai que l'artisan, et particulièrement le compagnon-cordonnier, demeure la principale victime de l'industrialisation, mais il ne fut pas une victime passive et résignée. 